\title{
An Unusual Cause of Cholestasis in an Infant: Biliary Atresia Type IIB
}

\author{
Poonam Sherwani $^{1}$ Rishi Bolia ${ }^{2}$ Ashish Kaushik ${ }^{1}$ Sumit Kumar ${ }^{1}$ Sanjeev Kishore ${ }^{3}$ Udit Chauhan ${ }^{1}$ \\ 1 Department of Radiodiagnosis, All India Institute of Medical \\ Sciences, Rishikesh, Uttarakhand, India \\ 2 Department of Pediatrics, All India Institute of Medical Sciences,

\begin{abstract}
Address for correspondence Poonam Sherwani, DNB, MNAMS, Department of Radiodiagnosis and Imaging, AlIMS, Rishikesh, Uttarakhand, India (e-mail: sherwanipoonam@gmail.com).
\end{abstract} Rishikesh, Uttarakhand, India

${ }^{3}$ Department of Pathology, All India Institute of Medical Sciences, Rishikesh, Uttarakhand, India

Indian J Radiol Imaging 2021;31:689-692.

\begin{abstract}
Biliary atresia (BA) is a progressive destructive cholangiopathy of unknown etiology that presents in early infancy. It has a worldwide frequency of 1:8,000-1:15,000 and is common in Asia than in the west. Based on the level at which the lumen of the

Keywords

- biliary atresia

- cholangiopathy

- common hepatic duct extrahepatic duct is obliterated, BA is classified into three types. Type III is the commonest $(\sim 85 \%)$ type and has the most proximal level of obstruction in the porta hepatis, while type II in which the atresia is at the level of the common hepatic duct, is the least common $(\sim 2.5 \%)$ and has been rarely reported. Here, we report the imaging features of an infant with type IIB biliary atresia.
\end{abstract}

\section{Introduction}

Biliary Atresia (BA) is an inflammatory progressive cholangiopathy that affects the intrahepatic and extrahepatic biliary tree. It is categorized into three types, of which type III in which the biliary tree is completely atretic is the most common. ${ }^{1,2}$ Type II is the rarest where the atresia is at the level of the common hepatic duct. Here, we describe a 4month-old infant who presented with cholestatic jaundice. Ultrasound and MRCP were suggestive of type IIB BA that was confirmed with a percutaneous transhepatic cholangiogram (PTC) and liver biopsy.

\section{Case History}

A 4-month-old female patient presented with complaints of jaundice with acholic stools and high colored urine since day 5 of life. On general examination, she was alert and had pallor and icterus. Abdominal examination showed firm hepatomegaly, splenomegaly, and moderate ascites. Laboratory investigation showed conjugated hyperbilirubinemia -total/direct bilirubin $(14.03 / 9 \mathrm{mg} / \mathrm{dL})$ and raised gammaglutamyl transpeptidase (GGT) activity $724(<40 \mathrm{IU} / \mathrm{mL})$. Serology for CMV (IgM) was negative.

Clinically, a possibility of a biliary obstructive pathology was considered and the child was evaluated. Ultrasound (LOGIQS8, GE, UK) was performed after withholding feeds for 3 to 4 hours using a curvilinear $(4-5 \mathrm{MHz})$ and a highfrequency linear transducer that revealed hepatomegaly $(9 \mathrm{~cm})$ with a coarsened echotexture and reduced echogenicity. There was a surface irregularity with a nodular outline, suggestive of chronic liver disease (CLD). There was moderate bilobar intrahepatic biliary radical dilatation (IHBRD) with inspissated secretions within the dilated biliary radicles. Gall bladder (GB) and common bile duct (CBD) were not visualized on repeated scans 24 hours apart. A triangular cord sign was evident (-Fig. 1C); however, a hepatic artery was not prominent. The subcapsular flow
DOI https://doi.org/ 10.1055/s-0041-1736165. ISSN 0971-3026.

\footnotetext{
(C) 2021. Indian Radiological Association. All rights reserved. This is an open access article published by Thieme under the terms of the Creative Commons Attribution-NonDerivative-NonCommercial-License, permitting copying and reproduction so long as the original work is given appropriate credit. Contents may not be used for commercial purposes, or adapted, remixed, transformed or built upon. (https://creativecommons.org/ licenses/by-nc-nd/4.0/)

Thieme Medical and Scientific Publishers Pvt. Ltd., A-12, 2nd Floor, Sector 2, Noida-201301 UP, India
} 


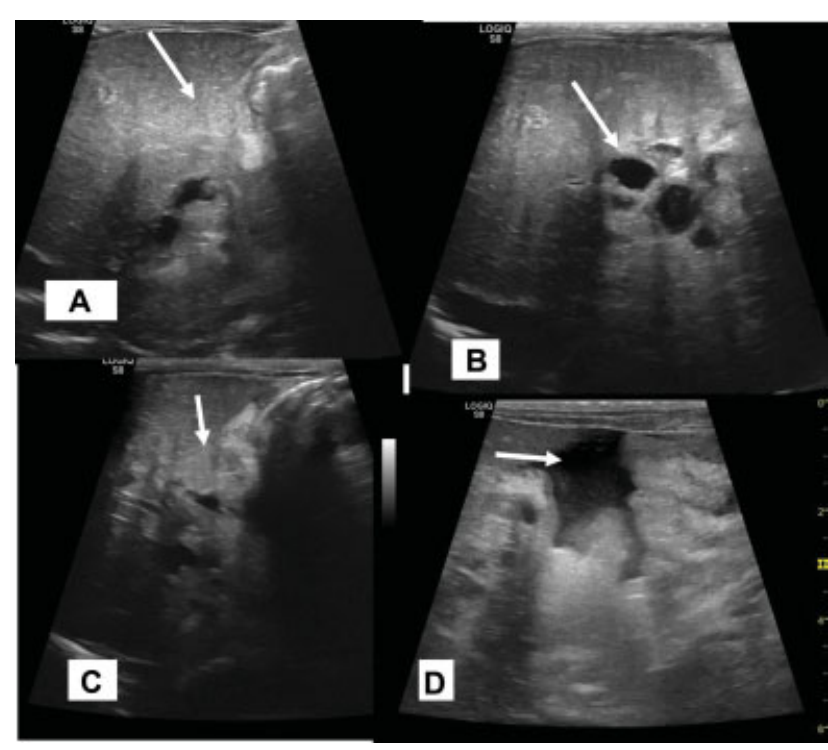

Fig. 1 (A-D) Transverse grayscale ultrasound using curvilinear and linear probes show nonvisualization of the gall bladder with empty $G B$ fossa (arrow in A). There were dilated biliary radicles in both lobes (arrow in B) with coarsened echotexture of the liver. There was increased echogenicity seen anterior to the portal vein, and CBD was not visualized at porta, which was suggestive of a triangular cord sign (arrow in C). Free fluid was also seen in the peritoneal cavity (arrow in D). CBD, common bile duct; $G B$, gall bladder.

was also not evident. The spleen was enlarged and there was free fluid in the peritoneal cavity ( - Fig. 1). MRI abdomen with MRCP revealed moderate central bilobar IHBRD with an abrupt cutoff at the level of the common hepatic duct (CHD). Primary confluence was patent. Cystic duct, CBD, and GB were not visible on MRCP. There was crossed fused ectopia, and the right kidney was lying on the left side below the left kidney (-Fig. 2).

As the child had features of decompensated liver disease, a surgical drainage procedure was not deemed feasible, and percutaneous transhepatic biliary drainage was done as a temporary measure to relieve jaundice through the right biliary system. Percutaneous transhepatic cholangiogram (PTC) revealed bilobar IHBRD with an abrupt cutoff at the CHD. Primary confluence was patent; however, there was no passage of contrast into distal CHD/CBD (-Fig. 3). As the stricture was not negotiable, the child was put on external drainage. In view of PTC findings, hepatobiliary iminodiacetic acid (HIDA) scan was not performed and a radiological diagnosis of BA type IIB was made. Subsequently, an ultrasound-guided liver biopsy was done. The portal tracts showed fibrous portal tract expansion and bile duct proliferation. There was the presence of intrahepatic and intracanalicular biliary cholestasis and portal to central fibrosis with nodule formation ( - Fig. 4). Features were suggestive of cirrhosis secondary to BA. Upper gastrointestinal endoscopy showed small esophageal varices and the major duodenal papilla was not visualized in the duodenum.

A liver transplant was planned. Unfortunately, while undergoing evaluation, the child developed spontaneous bacterial peritonitis and died of severe sepsis.

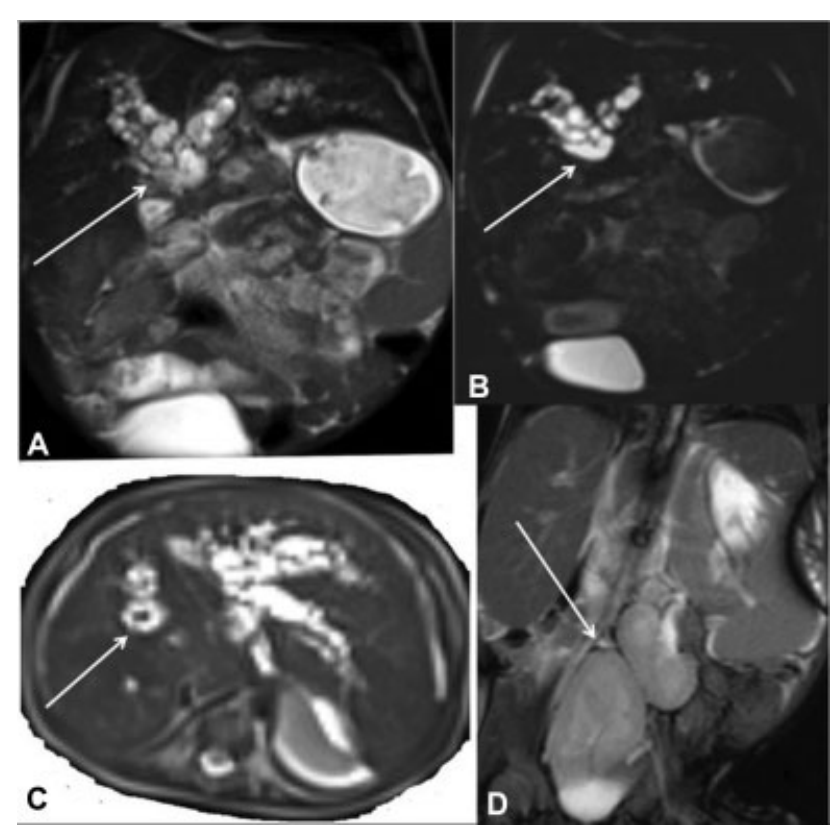

Fig. 2 (A-D) T2W FS coronal image showing bilobar intrahepatic biliary radicles dilatation (arrow in $\mathrm{A}$ ) with patent primary confluence and abrupt cutoff at the level of the common hepatic duct (arrow in B). Cystic duct, common bile duct, and gall bladder are not visualized. T2 hypointense inspissated secretions/plugs are seen in the dilated biliary radicles (arrow in C). Crossed fused renal ectopia is also seen. The right kidney is seen on the left side at the inferior aspect of the left kidney which is also malrotated (arrow in D). T2W FS, T2-weighted fat saturated.
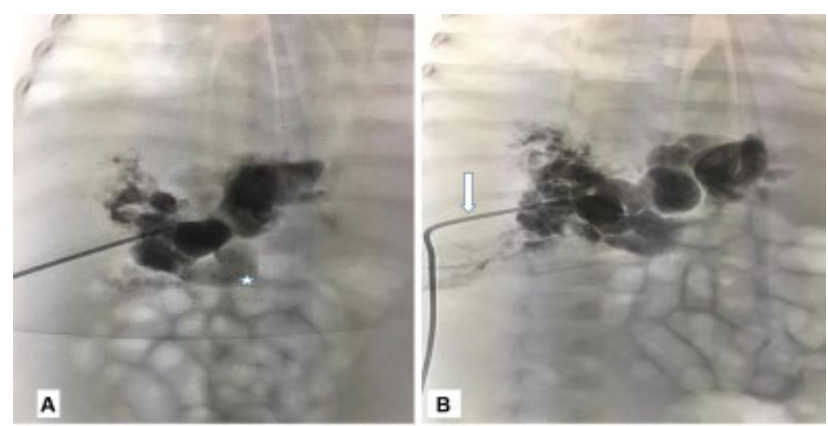

Fig. 3 (A) Percutaneous cholangiogram using the right ductal approach showing patent primary confluence with dilated bilobar ducts and abrupt cutoff at the CHD level $\left({ }^{*}\right)$. No passage of contrast seen in distal CHD, CBD, or duodenum. (B) An external drainage catheter was inserted for decompression and drainage of the biliary system (arrow). CBD, common bile duct; CHD, common hepatic duct.

\section{Discussion}

BA is a rare congenital cause of biliary obstruction. The etiology is still elusive although several viral, genetic, and immune-mediated theories have been postulated. ${ }^{2}$ There is inflammatory damage to both intrahepatic and extrahepatic bile duct leading to sclerosis, narrowing, and complete obliteration of the biliary tree, which leads to progressive fibrosis of the liver and eventually cirrhosis as seen in our child. The clinical presentation consists of jaundice, acholic stools, and dark urine, and hepatomegaly. They generally 


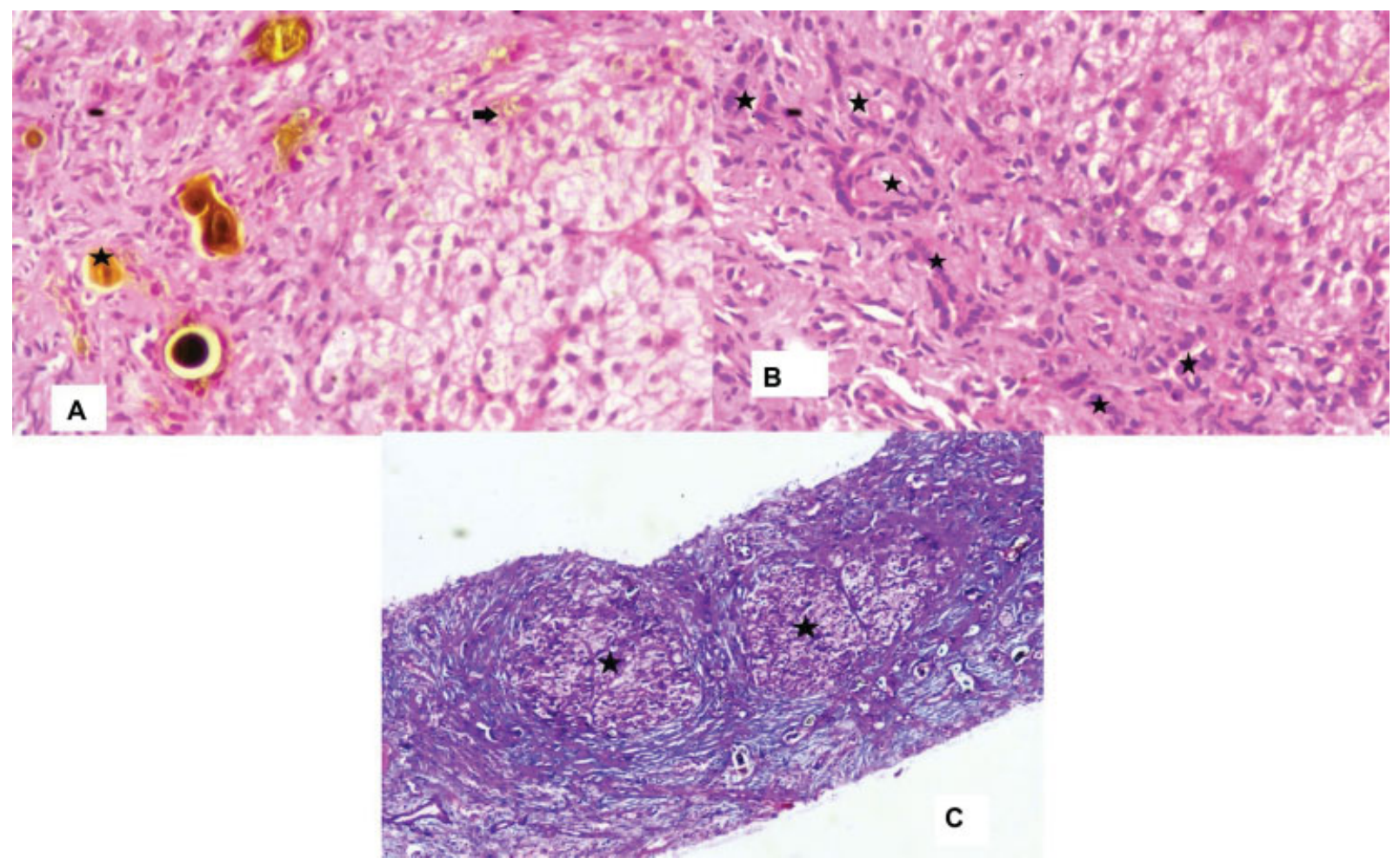

Fig. 4 (A) Microphotograph showing hepatocytes with ballooning. Degeneration (triangle). Intracanalicular (star) and intrahepatic (arrow) cholestasis is also noted. (HE x400). (B) Microphotograph showing bile duct proliferation (star). (HE x400). (C) Magnified view showing nodule formation (arrows), with surrounding fibrosis (Masson's trichrome $\times 100$ ). HE, hematoxylin and eosin.

appear well initially and have normal growth despite their jaundice, which leads to a delayed referral as seen in our child. Laboratory analysis includes direct hyperbilirubinemia and elevated GGT. ${ }^{3}$

Two forms of BA, syndromic or biliary atresia splenic malformation $(\sim 10 \%)$, associated with other congenital anomalies and non-syndromic ( $\sim 90 \%)$ in which BA is the isolated anomaly are described. ${ }^{2}$ Our child had associated crossed fused ectopia of the kidneys along with BA.

The Japanese Association of Pediatric Surgeons is the most widely used classification of BA, which is based on the level of atresia and is as follows. ${ }^{4}$

Type I: Atresia at the level of CBD (12\%), also known as distal BA. Gall Bladder and hepatic ducts are patent. Type II: Atresia at the level of CHD (2.5\%)-Rarest form. Type 2 is divided into IIA and IIB. CBD, cystic duct, and GB are patent in Type IIA, while obliterated in Type IIB.

Type III: Atresia at the porta hepatis (85\%), also known as complete BA.

\section{Diagrammatic Representation of Classification of EHBA}

The described infant was Type IIB BA, which is the rarest. The most commonly encountered BA is complete or Type III BA in which there is non-visualization of bile ducts and cystic duct and gall bladder. In other subtypes, bile ducts and gall bladder can be seen depending on the level of atresia. Due to limited literature available on other subtypes, the status of biliary radicles proximal to the atresia in other types is not described previously. In our patient, intrahepatic biliary radicles were dilated, so other differentials such as congenital stricture or web and inspissated bile duct syndrome were also considered.

Ultrasound is the preliminary investigation and requires fasting for at least 6 hours. Depending on the type, numerous sonographic findings are seen that include either nonvisualization or rudimentary GB, absence of contraction after feeding, and presence of triangular cord sign in the vicinity of the portal vein in type III BA ${ }^{5} \mathrm{~GB}$ is visualized in type I and IIA. Ultrasound will also detect the changes of CLD and portal hypertension as is seen in our case. Color Doppler findings include dilated hepatic artery and increased subcapsular flow that aid in the diagnosis. ${ }^{6}$ Our case showed IHBRD with nonvisualization of $\mathrm{CBD}$ and $\mathrm{GB}$ and superimposed CLD, suggesting type IIB BA.

MRI of the abdomen with MRCP is done to confirm the findings, for better depiction of biliary anatomy, level of atresia, as well as to look for any other associated congenital anomaly. The sensitivity of MRCP is 90 to $100 \%$ and specificity is 77 to $96 \%{ }^{7}$ Nonvisualization of the extrahepatic biliary tree and GB is the diagnostic hallmark of BA; however, the demonstration of only a normal CBD also does not necessarily exclude $\mathrm{BA}$, as the disease may involve only the proximal extrahepatic biliary system. In our patient CBD, cystic duct and gall bladder were not visualized, and there were dilated biliary radicles with T2 hypointense-filling defects, suggestive of inspissated secretions. These imaging features were consistent with a diagnosis of type IIB BA, which was confirmed by the percutaneous transhepatic cholangiogram 


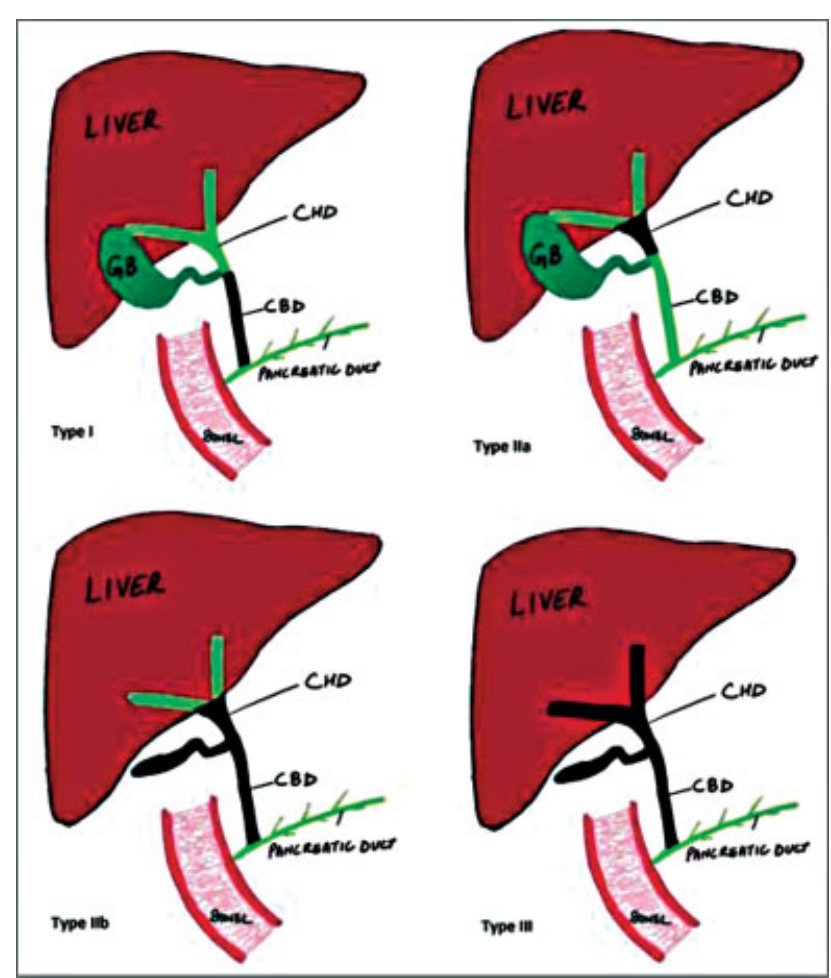

Fig. 5 Diagrammatic representation of the classification of biliary atresia.

(PTC) that demonstrated moderate dilatation of bilobar biliary radicles with a patent primary confluence. Just distal to the formation of CHD, there was an abrupt cutoff with non-passage of contrast distal to it. In children, inspissated bile duct syndrome may often mimic BA, and PTC cannot only help in establishing the diagnosis by showing ductal filling defects but an attempt at therapeutic lavage also can be made. ${ }^{8}$ A congenital stricture or web of the common hepatic duct is another rare condition that may present with features of biliary obstruction; however, the early clinical presentation and aggressive clinical course with features of established cirrhosis by 4 months of age would make it unlikely. An absent GB with non-visualization of the web, which is seen as a linear-filling defect on MRCP ruled out the diagnosis of the congenital web on imaging. ${ }^{9}$ The diagnosis of BA was further corroborated by the liver biopsy findings, which had a diagnostic accuracy of $90.1 \%$ for BA. ${ }^{10}$ A Kasai portoenterostomy is the treatment of choice; however, in children who present late with features of advanced cirrhosis, as seen in our child, one has to proceed directly for liver transplantation. We planned for the same but unfortunately, the child died of sepsis while undergoing evaluation for the same (-Fig. 5).

To conclude, we describe the multimodality imaging features of a child with Type IIB BA which is the rare type. Various imaging findings of Type III EHBA, which is the commonest type, are described previously; however, the imaging findings of type IIB have not been described previously to the best of our knowledge.

\section{Funding \\ None.}

\section{Conflict of Interest}

None declared.

\section{References}

1 Nio M. Japanese biliary atresia registry. Pediatr Surg Int 2017;33 (12):1319-1325

2 Sinha CK, Davenport M. Biliary atresia. J Indian Assoc Pediatr Surg 2008;13(02):49-56

3 Shankar S, Bolia R, Foo HW, et al. Normal gamma glutamyl transferase levels at presentation predict poor outcome in biliary atresia. J Pediatr Gastroenterol Nutr 2020;70(03):350-355

4 Kang LH, Brown CN. Pediatric biliary interventions in the native liver. Semin Intervent Radiol 2016;33(04):313-323

5 Lee MSet al. Radiology, 2009; Zhou L et al., Radiology 2015 and Zhou L et al., AJR 2016

6 Lee SM, Cheon JE, Choi YH, et al. Ultrasonographic diagnosis of biliary atresia based on a decision-making tree model. Korean J Radiol 2015;16(06):1364-1372

7 Yang JG, Ma DQ, Peng Y, Song L, Li CL. Comparison of different diagnostic methods for differentiating biliary atresia from idiopathic neonatal hepatitis. Clin Imaging 2009;33(06): 439-446

8 Davenport M, Betalli P, D’Antiga L, Cheeseman P, Mieli-Vergani G, Howard ER. The spectrum of surgical jaundice in infancy. J Pediatr Surg 2003;38(10):1471-1479

9 Papaziogas B, Lazaridis C, Pavlidis T, Galanis I, Paraskevas G, Papaziogas T. Congenital web of the common bile duct in association with cholelithiasis. J Hepatobiliary Pancreat Surg 2002;9 (02):271-273

10 Russo P, Magee JC, Anders RA, et al; Childhood Liver Disease Research Network (ChiLDReN) Key histopathologic features of liver biopsies that distinguish biliary atresia from other causes of infantile cholestasis and their correlation with outcome: a multicenter study. Am J Surg Pathol 2016;40(12):1601-1615 